\begin{tabular}{lc}
\hline Material & $\begin{array}{c}\text { Ultimate Tensile Strength } \\
\left(\mathrm{MN} / \mathrm{m}^{2}\right)\end{array}$ \\
\hline Silicate glass fibre & 1400 \\
Crystalline steel wire & 2000 \\
$\mathrm{Pd}_{80} \mathrm{Si}_{20}$ glass & 1360 \\
$\mathrm{Cu}_{60} \mathrm{Zr}_{40}$ glass & 2000 \\
$\mathrm{Ni}_{75} \mathrm{Si}_{8} \mathrm{~B}_{17}$ glass & 2700 \\
$\mathrm{Co}_{75} \mathrm{Si}_{15} \mathrm{~B}_{10}$ glass & 3000 \\
$\mathrm{Fe}_{80} \mathrm{~B}_{20}$ glass & 3200 \\
$\mathrm{Fe}_{78} \mathrm{Si}_{10} \mathrm{~B}_{12}$ glass & 3400 \\
\hline
\end{tabular}

glass specimen having the same shape can withstand a local plastic shear strain well in excess of $50 \%$. One obvious application that would capitalize on these qualities is their use as fibres for reinforcement, for example in automobile tyres. Not surprisingly, the mode of deformation exhibited by glassy metals is quite different from that observed in crystalline metals. The inherent weakness of a crystal lies in its ability to be readily dislocated. The virtually un-avoidable presence of dislocations is responsible for the fact that the actual strength of a crystal is seldom more than one-thousandth of the ideal value. It takes surprisingly little force to move a dislocation, and stresses are thus unable to build up to potentially dire levels.

The lack of translational symmetry in the glass precludes a deformation mechanism based on normal dislocation movement, and this is why covalent glasses are always brittle. Remarkably, metallic glasses nevertheless appear to fail by ductile rupture, and the mechanism by which this occurs has understandably aroused interest. In 1972, H.S. Chen, H.J. Leamy, and T.T. Wang observed surface striations on a deformed specimen, suggesting some sort of shear band arrangement. P. Donovan went on to show that if the surface of such a specimen is polished and then etched, the striations reappear, indicating that the sheared zone has been modified by the shearing process. D.E. Polk and David
Turnbull explained this on the basis of alteration of the compositional short-rang $\epsilon$ order, caused by the shear movements. Fractured surfaces of glassy metals display a characteristic vein or ridge pattern, this being reported by Turnbull and F. Spaepen, and independently by C.A. Pampillo and R.C. Reimschuessel. S. Takayama and R. Maddin compared such markings with those formed when two smooth surfaces held together with grease are separated.

The glassy-metal story is still in its infancy but it is already clear that this new type of material is here to stay. The next few years will no doubt bring a mushrooming of new compunds and new applications. Whether or not these developments will be matched by significant advances on the scientific front is perhaps more debatable. One thing at least is assured: glassy metals will continue to be the focus of much attention in university and industrial laboratories.

\title{
The Surfaces of Metals
}

\author{
Derek J. Fabian, Glasgow \\ University of Strathclyde
}

Metal surface physics is fast becoming a field of major significance. Advancements in the physics of surfaces and interfaces generally, especially in connection with materials degradation, have important implications for all of modern technology, from electronic and microprocessor engi-

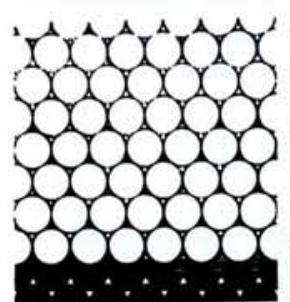

FCC (111)

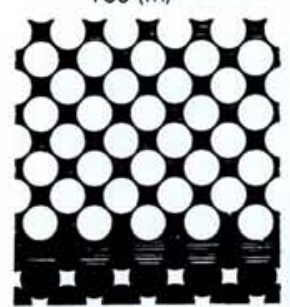

FCC (100)

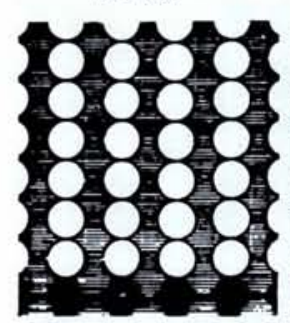

FCC (110)

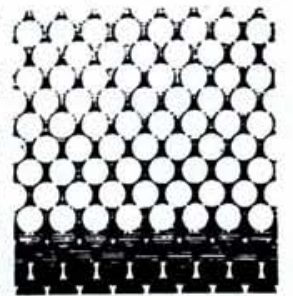
BCC (110)

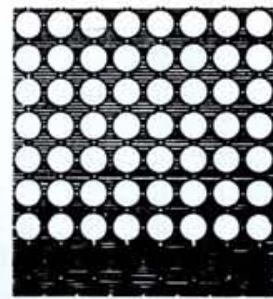

BCC (100)

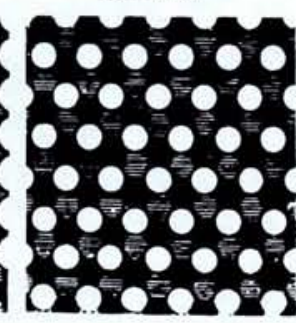

BCC (111)

Fig. 1 -Ideal surface-atom arrangements formed by the high-density planes of face-centred cubic and body-centred cubic metals. neering where semiconductor physicists move constantly to smaller devices, through chemical engineering where chemists and metallurgists continuously seek - for example - an improved understanding of e.g. catalysis, to mechanical engineering where a more thorough knowledge of both the physics and chemistry of processes such as corrosion, oxidation, embrittlement, friction, wear and adhesion is needed, as components become subjected to more extreme conditions. Surface treatments and coatings, and an understanding of thin-film adhesion and surface modification, are already paramount to the further development of many technologies.

\section{Classification of Metal Surfaces}

Surfaces are sometimes sub-divided scientifically into "pure", "clean" and "real" (or "technical"). A pure surface is one in which the metallic lattice is in atomic contact with its adjoining gas (or liquid) phase, and is uncontaminated by adsorbed species. A clean surface might have, for example, a thermodynamically stable thin oxide film, but is free from otherwise adsorbed foreign species. A real or technical surface, more commonly encountered in engineering applications, is one that carries an oxide film plus adsorbed or deposited sometimes embedded - foreign material.

To produce a pure surface, the metal crystal must either be prepared or cleaved in ultra high vacuum, preferably of the order of $10^{-10}$ Torr. A monolayer of contamination, oxide for example, will form on a pure surface in $1 \mathrm{~s}$ when exposed to $10^{-6}$ Torr of residual gas. The resulting metal surface would be classed as "clean". Studies of the more typical metal surfaces found in industrial practice, sometimes

Fig. 2 - Terrace-Ledge-Kink (TLK) model representation of a metallic crystal surface.

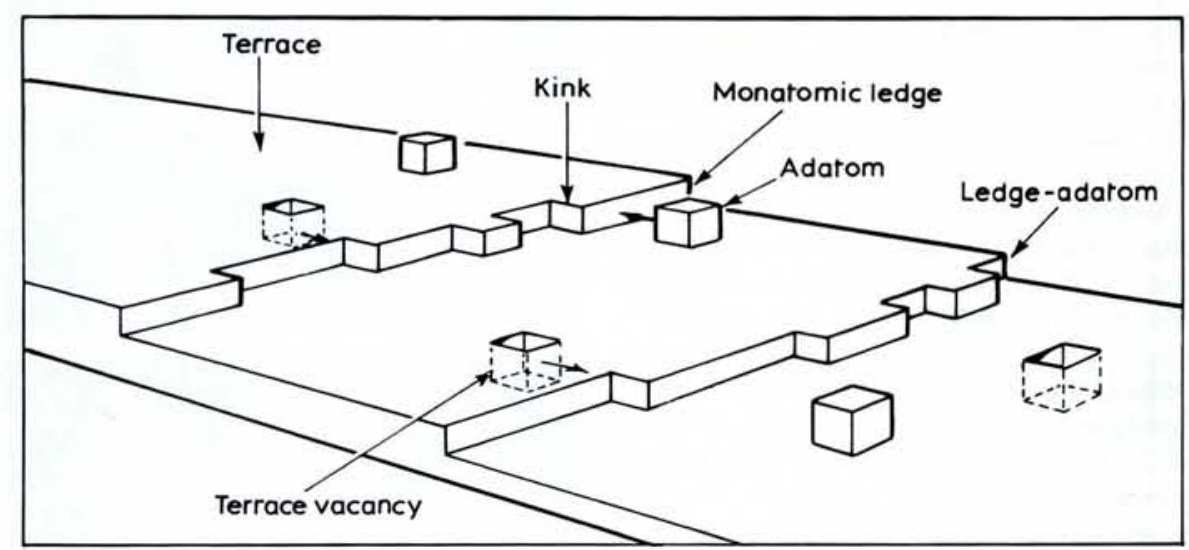




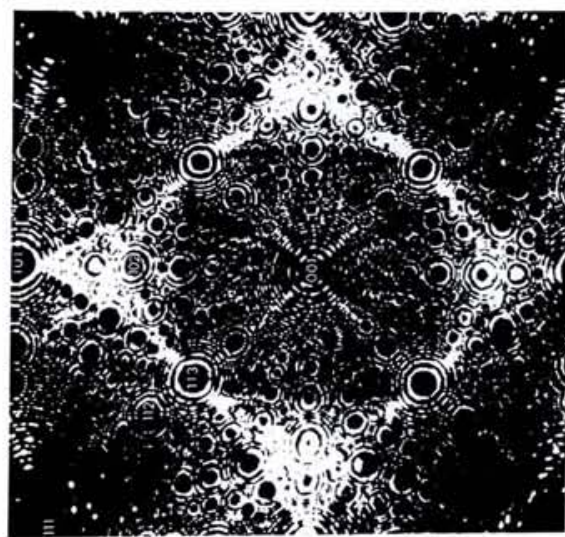

Fig. 3 - Field-ion micrograph image of the atomic layers formed at a fine tip $1 \sim 10^{-8} \mathrm{~m}$ radius of curvature) of platinum metal.

suitably passivated, coated or modified, even deliberately "corroded" or activated, form a physical science which - because of the importance of developing relationships of surface condition with, for example, friction and wear - is just as far reaching as the study of surface atomic structure.

\section{Pure Metal Surfaces}

The whole of our physical and chemical understanding of the metallic surface is centred around the fact that the surface atoms of a crystalline material exist in a special environment with respect to their neighbouring atoms; different from that of an atom in the bulk of the crystal. There is a huge asymmetry in the field of force to which they are subject, resulting in a higher energy state, lower coordination to near neighbours, and considerably increased reactivity towards foreign atoms or molecules because of their chemical tendency to saturate missing bonds and establish a symmetrical crystal field.

If a metal crystal of ideal structure is cleaved through a plane orientated in relation to one of its crystallographic axes, then two identical surfaces are created. These surfaces can be considered "ideal" if there is no relaxation process and all the surface atoms remain in the crystallographic positions they occupied initially. The "crystal" structures of such surfaces, for high atomic density planes, are illustrated in Fig. 1 for bcc and fcc metal crystals.

It is immediately clear how the number of nearest neighbour atoms is reduced, and by different amounts for different crystal faces; from 12 in the bulk of fcc metal, for example, to 9,8 and 6 respectively for the (111), (100) and (110) faces of the crystal. This gives the surface atoms considerable reactivity towards foreign neighbouring atoms or molecules, a reactivity that varies with crystallographic orientation of the surface. The influence of surface orientation is also reflected in differences in physical properties, such as surface energy, work function and optical reflectivity.
Pure surfaces can of course be formed at an angle to one or more of the basic crystallographic planes leading to a sub-classification of pure surfaces into: (a) surfaces of high atomic density with minimum surface energy: (b) surfaces slightly mis-orientated to the high-density planes which can be represented theoretically by a statistical model; (c) complex surfaces which have a "natural" roughness and high surface energy.

The model that best describes surfaces slightly mis-orientated was introduced by Kossel and Stranski and is known as the terrace-ledge-kink, or TLK model, see Fig. 2. The crystal surface is represented as a succession of high-density "ledges", joined by steps or "terraces" containing a specific distribution of "kinks", which provide curvature to the terraces. Each of the steps will have a free energy per unit length, which must be added to the surface free energy calculated from nearest neighbour interactions for pure high density planes. This extra free energy and thus the total surface free energy will be proportional to the angle of mis-orientation.

From the lowest temperatures upwards, the formation of surface vacancies and the self-adsorption of ad-atoms are responsible for a natural roughness of the surface. However, provided their energies of formation are known, the surface concentration of such vacancies and ad-atoms may be evaluated on a statistical basis, and calculations indicate that high density planes should remain atomically "smooth" up to temperatures near the melting point of the crystal. The validity of the model, and the presence of terraces, ledges and even of kinks on an atomic scale, has been confirmed by field-ion microscopy (Fig. 3), while the stability of high density planes in the absence of contamination - can be deduced from low-energy electron diffraction (LEED) measurements.

The model also predicts, from calculations of the relaxation expected as a result of the asymmetrical field for the surface atoms, significant expansions of metal lattices perpendicular to the surface - ranging from $15-20 \%$ between the first two layers to $2-5 \%$ between the second and third layers - but this has not been confirmed experimentally. The calculations are made difficult by uncertainties in the choice of interaction potential and unavoidable arbitrary extrapolation from potentials and constants effective in the bulk to those pertaining at the surface.

Theoretical Models of Surface Adsorption

Despite the range and variety of experimental techniques for studying adsorbed species, their distribution in terms of the atomic topography of the substrate metal is difficult to determine. Recourse to theoretical models is necessary and, although these can be shown to be consistent with
Eight two- to four-day seminars on advanced optical/electro-optical technology.

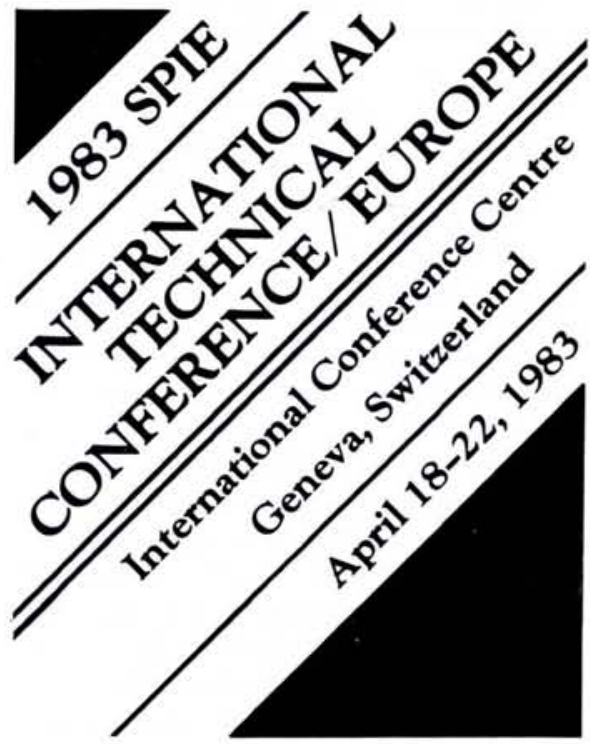

- Advances in Laser Scanning and Recording

- Advanced Infrared Sensor

Technology

- Applications of Digital Image Processing

- Industrial Applications of Laser Technology

- Optical System Design, Analysis, and Production

- Thin Film Technologies

- Three-Dimensional Imaging

- New Optical Materials

Approximately 300 papers will be presented, in addition to fourteen tutorials on related topics, and an international instrument display.

Sponsored by SPIE-The International Society for Optical Engineering.

COOPERATINGSPONSORS: Association $\mathrm{Na}$ tionale de la Recherche Technique; Battelle-Geneva Research Centres; Comité Francais d'Optique; European Optical Committee (representing National Optical Committees); European Physical Society; German Society for Applied Optics; IEEE Computer Society; Italian Electronic and Electrotechnical Association; Swiss Federal Institute of Technology in Lausanne; Swiss Society for Optics and Electron Microscopy.

FOR ADDITIONAL PROGRAM AND REGISTRATION INFORMATION: Contact SPIE, P.O. Box 10 , Bellingham, WA 98227-0010 USA, telephone (206) 6763290, telex 152556 SEA. In Europe, contact the European Physical Society, P.O. Box 69, CH-1213 Petit-Lancy 2 Switzerland, telephone (022) 93-11-30, telex 23-455 (alarm ch). FOR EXHIBIT INFORMATION: Contact the U.S. offices of SPIE. In Europe, contact Vicki BASTIEN, rue du Poulain, Villey-St. Etienne, 54200 Toul, France, telephone (8) 343-9461, telex 850078 (subscriber No. 85) 
data obtained from LEED measurements, as models they remain largely speculative.

It is fairly clear that in the initial stages of adsorption, the atoms or molecules tend to localize at specific surface "sites" where interaction with the substrate atoms is strongest. The most reactive sites for a specific species are usually those in which it makes contact with the maximum number of surface atoms of the metal substrate. Adsorption on such a site will then inhibit adsorption at adjacent sites and a periodic arrangement of adsorbed atoms (or molecules) will result, with a unit cell which is simply related to that of the substrate surface.

Adsorption may go beyond this first stage, but requires an activation energy for the adsorbed atoms to move from their electronic potential wells and to regroup into more compact arrangements. Predicted stages in the adsorption process can be observed in carefully conducted heat-ofadsorption measurements, and such studies have been particularly successful for example in the controlled adsorption of carbon monoxide on different single crystal faces of metallic nickel.

The whole concept of surface adsorption "sites" arises from considerations of atomic bonding and co-ordination, and raises important theoretical questions concerning the electronic energy state of an adsorbed species. Conflicting models occur: one in which the substrate metallic valence band is maintained "rigid" during electronic attachment of a species to the surface (the rigid band model), and an alternative view in which a surface molecule is formed between a substrate atom and a surface atom, with considerable perturbing effect on the metal valence band by the adsorbed species. A more recent and probably more correct model introduced by Soven and Schrieffer, is one invoking a local density of states (LDS) manifest around surface atoms of a metal (Fig. 4e), which can interact quantum mechanically with an adsorbate atom core-level (Fig. 4a) to give either a weak interaction (Fig. 4b) where a rigid band model would apply, or a strong interaction (Fig. 4d) producing bonding

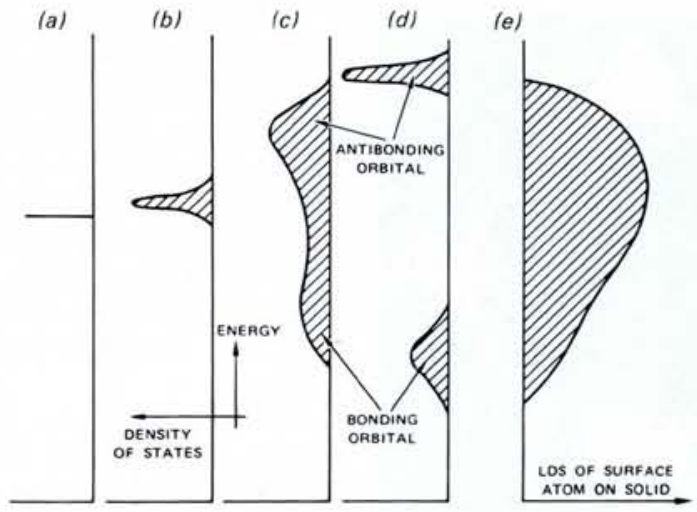

Fig. 4 - Theoretical model for the interaction of a substrate surface-atom local density of electron states (e) with an adsorbed atom electronic core-level (a). A strong interaction (d) gives rise to bonding and antibonding surface states. (Reproduced from Morrison S.R., "The Chemical Physics of Surfaces" (Plenum Press, London) 1977.)

and anti-bonding orbitals consistent with a surface molecule picture.

The local density of states, when an intermediate interaction occurs, is changed to a form of a kind illustrated in Figure 4c.

A possible test of the LDS model could come from soft $X$-ray emission studies of surface species. This technique, used extensively (e.g. by the author and coworkers) for LDS in the bulk electronic structure of metals and alloys, could be made surface sensitive by using low excitation energy and also glancing angle of excitation beam; the considerable loss of intensity that these would cause (and the technique already suffers from inherent low intensity spectra) could be overcome by using synchrotron radiation to produce the spectra by fluorescence excitation. The advent of synchrotron radiation has already made possible the study of extended X-ray adsorption fine structure (EXAFS) spectra from surface molecules, with the aim of providing information on their bonding environment.

\section{Coating of Metal Surfaces}

Industrial applications of surface metal physics is centred mostly around the coating of metals, for improvement of their resistance to corrosion and wear, plus the formation of active catalysts for use in chemical processes. The most recent and rapidly developing methods for producing coatings with desirable properties are ionimplantation and reactive plasma treatment. These fields overlap, but in each, over the past 5-10 years, the advancements and the increase in number of industrial applications have been enormous.

This has especially been so in reactive plasma treatment, which provides a unique method for conducting high-temperature chemistry at low temperatures; it is valuable therefore in producing such coatings as $\mathrm{TiN}$ and $\mathrm{SiC}$ on substrate materials that could not sustain the temperatures required for the more usual chemical vapour deposition. But there remains much fundamental physics still to be understood in both plasma (or glow-discharge) and ionbombardment treatments of metal surfaces. Plasma deposited coatings sometimes display unexpected properties, with evidence of modifications to the surface that are not fully understood.

Almost all of the surface experimental techniques available today, especially the spectroscopic methods, can be brought into play in this area of investigation. And in some industrial reactive plasma deposition processes, almost nothing is known, for example, about the transient species involved, nor of the mechanism of deposition. This area alone offers opportunities for exciting physics.
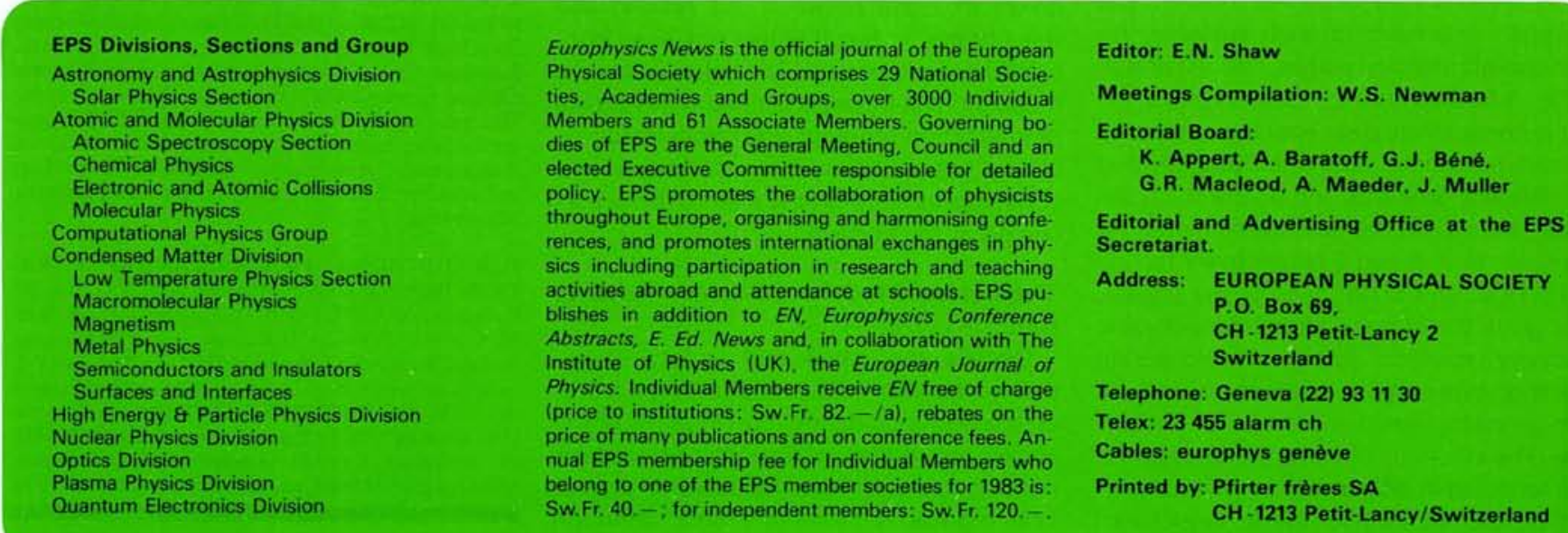